\title{
Parâmetros Físico-Químicos, Teor de Fibra Bruta e Alimentar de Pós Alimentícios Obtidos de Resíduos de Frutas Tropicais
}

\author{
Ana Maria Athayde Uchoa ${ }^{1}$, José Maria Correia da $\operatorname{Costa}^{1}$, Geraldo Arraes Maia ${ }^{1}$, Elisabeth \\ Mary Cunha Silva ${ }^{1}$, Ana de Fátima Fontinele Urano Carvalho ${ }^{2}$, Tatyane Ribeiro Meira ${ }^{1}$
}

As frutas tropicais são utilizadas como matéria-prima para a fabricação de diversos produtos alimentícios, destacando-se mais recentemente a polpa de fruta congelada de ampla aceitação entre os consumidores, utilizada para a elaboração de sucos e refrescos comercializados em lanchonetes, escolas, restaurantes, além do consumo doméstico. Atualmente sabe-se que a fibra alimentar desempenha no organismo funções importantes como intervir no metabolismo dos lipídios e carboidratos e na fisiologia do trato gastrointestinal, além de assegurar uma absorção mais lenta dos nutrientes e promover a sensação de saciedade. Estudos mais recentes também comentam sobre as ações benéficas da fibra alimentar em doenças respiratórias e função pulmonar. Em função do exposto, este trabalho teve como objetivo determinar os parâmetros físico-químicos e o teor de fibra bruta e fibra alimentar dos resíduos do bagaço do caju, bagaço da goiaba e casca do maracujá, obtidos do processamento de polpa de fruta e transformados em pós alimentícios. Dentre os parâmetros físicoquímicos analisados foi verificado que os pós alimentícios dos resíduos de caju, goiaba e maracujá apresentam em predominância: ${ }^{\circ}$ Brix $\left.40,48 \pm 0,00 ; 10,48 \pm 0,00 ; 20,56 \pm 0,00\right)$; açúcares redutores $(36,55 \pm 0,65$; $5,31 \pm 0,13 ; 8,3 \pm 0,10 \%)$; vitamina C $(34,72 \pm 0,29 ; 21,55 \pm 3,66 ; 11,76 \pm 0,13 \mathrm{mg} / 100 \mathrm{~mL})$; fibra bruta $(9,92 \pm 1,28$; $39,56 \pm 0,46 ; 26,31 \pm 1,09 \%)$; fibra alimentar $(3,26 \pm 0,01 ; 24,46 \pm 3,73 ; 17,07 \pm 0,79 \%)$; A partir dos resultados obtidos pode-se considerar que os pós alimentícios destes resíduos são boas fontes de vitamina $\mathrm{C}$, açúcar redutor e apresentam altos teores de fibra bruta e alimentar.

Palavras-chave: Resíduos de frutas; pós alimentícios; fibra alimentar; utilização de subprodutos

\section{Physicochemical Parameters and Crude and Dietary Fiber Content of Edible Powders From Tropical Fruit Residues}

Tropical fruits can be used as raw material for the manufacture of a variety of food products most notably frozen fruit pulps, which have deserved special attention due to their wide acceptance among consumers. They have been used in the formulation of juices and commercial refreshments sold in snack bars, schools, restaurants, besides the direct home consumption. Dietary fiber is known to play such important functions in the body as modulating lipid and carbohydrate metabolism and guaranteeing the proper gastrointestinal physiology. More recent studies also comment on the beneficial action of dietary fiber in respiratory illnesses and pulmonary function. Additionally, it assures a slower absorption of nutrients and signals satiety, thus counteracting obesity. This work was carried out to determine the physicochemical characteristics of the total

\footnotetext{
${ }^{1}$ Departamento de Tecnologia de Alimentos - CCA/UFC.

${ }^{2}$ Departamento de Biologia, CC/UFC.
} 
and dietary fiber content of the residues of cashew and guava bagasse and passion fruit rind obtained from pulp processing of frozen fruits transformed into edible powders. The powders of the cashew, guava and passion fruit residues were found to be rich in: total soluble solids ${ }^{\circ}$ Brix $(40.48 \pm 0.00 ; 10.48 \pm 0.00 ; 20.56 \pm$ $0.00)$; reducing sugars $(36.55 \% \pm 0.65 ; 5.31 \% \pm 0.13 ; 8.3 \% \pm 0.10)$; vitamin $\mathrm{C}(34.72 \pm 0.29 ; 21.55 \pm 3.66$; $11.76 \pm 0.13 \mathrm{mg} / 100 \mathrm{~mL})$; raw fiber $(9.92 \% \pm 1.28 ; 39.56 \% \pm 0.46 ; 26.31 \% \pm 1,09)$ and dietary fiber $(3.26 \% \pm$ $0.01 ; 24.46 \% \pm 3.73 ; 17.07 \% \pm 0.79)$, respectively. From the results it can be concluded that powders of these residues are nutrient-rich and may be considered as good sources of vitamin $\mathrm{C}$, reducing sugars and offer high contents of total and dietary fibers.

Key words: Fruit residues; nourishing powders; dietary fiber; byproduct utilization

\section{Introdução}

A importância econômica da fruticultura para as diversas regiões do Brasil não pode ser medida apenas através de dados estatísticos. O segmento está entre os principais geradores de renda, de empregos e de desenvolvimento rural. Os excelentes índices de produtividade e os resultados comerciais obtidos nas últimas safras são fatores que demonstram a vitalidade deste setor, que veio para ficar e para se desenvolver (Beling [4] e Embrapa [10]).

A fruticultura mundial foi responsável pela produção de 504,966 milhões de toneladas em 2005, sendo o Brasil o terceiro maior produtor de frutas, com uma produção acima de 35 milhões de toneladas, cerca de $5 \%$ da mundial, ficando atrás apenas da China e da Índia (FAO [12]). Deste total, apenas 2,3\% da colheita é exportado, o que corresponde a 827.708 toneladas (ANUÁRIO [3]). O Nordeste brasileiro possui condições climáticas que proporcionam ampla variedade de frutas tropicais. A conversão destas frutas em sucos, polpas, geléias e compotas torna possível a utilização do excedente destas frutas frescas no desenvolvimento de outros produtos alimentícios (Oliveira et al. [19]).

O cajueiro é cultura de grande importância econômica, tanto pelo fato de seus frutos serem consumidos in natura como pela sua industrialização, resultando em sucos e outros produtos bastante consumidos nos mercados interno e externo. Atualmente, é cultivado em diversos países, destacando-se Índia, Brasil, Moçambique e Tanzânia (Petinari et al.[22]). Associado ao caráter social e econômico do cajueiro, existe ainda a característica de tolerância à seca, credenciando-o como uma espécie capaz de gerar riquezas e ser necessário para fixar o homem no campo (SEAGRE [25]).

A goiaba é uma das frutas de maior importância nas regiões subtropicais e tropicais, não só devido ao seu elevado valor nutritivo, mas pela excelente aceitação do consumo in natura, além de ser apreciada por suas características de sabor e aroma e elevado valor nutritivo. A goiaba contém quatro vezes mais vitamina $\mathrm{C}$ do que a laranja e quatro vezes mais cálcio do que o tomate. Além disso, é rica em fibras, vitamina $\mathrm{E}$, e apresenta o dobro da quantidade de licopeno presente no tomate (Silva [27]).

O maracujá, originário da América tropical, é largamente cultivado e processado em todo o mundo. Esta fruta tem sido bastante consumida, principalmente como suco, devido ao seu aroma e acidez acentuados, e também em uma série de outros produtos como sorvetes, mousses e bebidas alcoólicas. $\mathrm{O}$ maracujá é rico em componentes fenólicos, que estão enquadrados na categoria dos fitoquímicos (Sandi et al. [24]).

No entanto, o processamento de frutas pelas indústrias gera grandes quantidades de resíduos, que podem ser perfeitamente utilizados no desenvolvimento de novos produtos alimentícios, aumentando seu valor agregado. É interessante também lembrar que o aproveitamento destes resíduos irá contribuir muito para a melhoria do meio ambiente, tendo em vista os grandes volumes produzidos pelas indústria e eliminados em locais inadequados. 
A fibra alimentar é uma fração complexa, composta por um conjunto de componentes, presentes nos alimentos vegetais, representados pela soma de lignina e polissacarídeos (celulose, hemicelulose, pectina, mucilagem e goma), sendo estes classificados, segundo sua solubilidade em água, como solúveis e insolúveis (Lobo e Silva [18]). Atualmente, a fibra alimentar é considerada alimento funcional, pois desempenha no organismo funções importantes como intervir no metabolismo dos lipídios e carboidratos e na fisiologia do trato gastrointestinal, além de assegurar uma absorção mais lenta dos nutrientes e promover a sensação de saciedade (Dutra e Marchini [9]).

A composição e as propriedades físico-químicas da fibra alimentar podem explicar a sua função nos alimentos. Essas informações podem ser aplicadas para a compreensão dos efeitos fisiológicos das fibras. Portanto, o estudo dos teores de fibra bruta e alimentar e das propriedades físicoquímicas dos resíduos do bagaço do caju, bagaço da goiaba e casca do maracujá é essencial para o desenvolvimento de novos produtos.

Em função do exposto, este trabalho teve como objetivo determinar os parâmetros físico-químicos e o teor de fibra bruta e alimentar dos resíduos do bagaço do caju, bagaço da goiaba e casca do maracujá, não aproveitados pelas indústrias de beneficiamento de frutas, visando uma melhor utilização

\section{Material e Métodos}

Os materiais utilizados para este experimento foram os resíduos de caju, de goiaba e de maracujá, obtidos do processamento de polpa de fruta congelada de uma indústria de polpa de fruta do Estado do Ceará. Os resíduos passaram, inicialmente, por uma secagem em estufa à vácuo, a temperatura de $65^{\circ} \mathrm{C}$, por um período de 16 horas, para serem transformados em pós alimentícios. Após a secagem, os resíduos foram triturados no liquidificador semi- industrial e, posteriormente, foram passados em um conjunto de peneiras de aberturas de 2,$0 ; 0,60 ; 0,42 ; 0,25 ; 0,18 ; 0,15$ e $0,07 \mathrm{~mm}$. O acondicionamento dos pós alimentícios foi realizado em vidros de $200 \mathrm{ml}$, previamente higienizados, envoltos por papel alumínio e tampados com filme plástico, para evitar a entrada de umidade, permanecendo a uma temperatura ambiente de $23 \pm 2^{\circ} \mathrm{C}$ até o momento das análises físico-químicas.Todo o processo de obtenção dos pós alimentícios seguiram os preceitos das Boas Práticas de Fabricação no Laboratório de Frutas e Hortaliças do Departamento de Tecnologia de Alimentos da Universidade Federal do Ceará.

As análises físico-químicas foram realizadas no Laboratório de Frutas e Hortaliças do Departamento de Tecnologia de Alimentos da Universidade Federal do Ceará e a análise de fibra alimentar no Laboratório de Biologia do Departamento de Biologia da Universidade Federal do Ceará. Todas as análises físico-químicas foram realizadas em triplicata. O conteúdo de ácido ascórbico foi determinado através do método titulométrico, baseado na redução do indicador 2,6diclorofenolindofenol pelo ácido ascórbico (Person e Cox [20]). As análises de cinzas foram realizadas seguindo as normas da A.O.A.C. [2] e as de proteínas seguiram a metodologia descrita pela A.A.C.C. [1], com a utilização da técnica micro-Kjeldahl. A análise de fibra bruta foi realizada submetendo as amostras à digestão ácida, com solução de ácido sulfúrico $1,25 \%$, seguida por digestão alcalina com hidróxido de sódio 1,25\% (Brasil [7]). Para determinação da fibra alimentar, utilizou-se o método enzimático gravimétrico (Prosky et al.[23]).

As análises de sólidos solúveis totais ( $\left.{ }^{\mathrm{B}} \mathrm{Brix}\right)$, determinados por refratometria, foram realizadas a partir de uma amostra de $10 \mathrm{~g}$ dos pós alimentícios em um béquer e a esta quantidade foi adicionado $100 \mathrm{ml}$ de água destilada e agitada por 30 minutos. Em seguida, colocou-se um pouco deste material em gaze e espremeu-se algumas gotas no refratômetro e fez-se a leitura direta no equipamento; a acidez total titulável foi verificada através de titulação com $\mathrm{NaOH} 0,1$ molar e os resultados expressos em 
percentagem de ácido cítrico; o $\mathrm{pH}$ foi determinado através de um medidor de marca Hanna Instruments. A umidade foi determinada em uma estufa à $105^{\circ} \mathrm{C}$ e os lipídios foram determinados em aparelho de extração Soxhlet, segundo Brasil [7]. Os açúcares redutores foram expressos em percentual de glicose e, para a determinação dos açúcares não-redutores, foi realizada uma inversão ácida prévia nos extratos das amostras e os resultados foram expressos em percentual de sacarose, de acordo com Brasil [7]. Os açúcares totais foram calculados a partir da somatória dos açúcares redutores e não-redutores, com as devidas correções para expressão dos resultados em termos de percentual de glicose.

\section{Resultados e Discussão}

Na Tabela 1, são mostrados os resultados das médias e desvio padrão dos parâmetros físico-químicos dos diferentes pós alimentícios. Podemos verificar que, apesar dos resíduos de bagaço de caju, bagaço de goiaba e casca de maracujá terem sido submetidos à secagem em estufa à vácuo a $65^{\circ} \mathrm{C}$, é possível observar ainda altas concentrações de vitamina $\mathrm{C}$ presentes nos pós alimentícios. Os teores de vitamina $\mathrm{C}$ encontrados nestes pós de caju, goiaba e maracujá foram de 34,$72 ; 21,55$ e 11,76 $\mathrm{mg} / 100 \mathrm{~g}$, respectivamente. Ao comparar os valores encontrados nestes pós com a ingestão diária recomendada para adultos (Brasil [5]), que estabelece um teor de $60 \mathrm{mg}$, observamos que todos os pós analisados podem ser considerados fontes importantes de vitamina C. A vitamina $\mathrm{C}$ é a mais facilmente degradável de todas as vitaminas, sendo estável apenas em meio ácido, na ausência de luz, de oxigênio e de calor. Provavelmente, as boas concentrações desta vitamina em nossos pós alimentícios estejam associadas aos cuidados que tomamos no acondicionamento das nossas amostras em recipientes de vidro coberto com papel alumínio e filme PVC, para evitar entrada de luz e oxigênio.

A acidez é um importante parâmetro na apreciação do estado de conservação de um produto alimentício. Os valores de acidez, encontrados nos pós dos resíduos de caju, goiaba e maracujá, variaram de 1,21 a $1,38 \mathrm{~g} / 100 \mathrm{~g}$. Felipe [13], analisando os mesmos pós, encontrou para o pó de resíduo de caju $1,36 \mathrm{~g} / 100 \mathrm{~g}$, para goiaba $0,97 \mathrm{~g} / 100 \mathrm{~g}$ e para maracujá $5,71 \mathrm{~g} / 100 \mathrm{~g}$. Observa-se que o valor da acidez para o pó de maracujá, encontrado pelo autor acima citado, é quatro vezes maior que o encontrado neste estudo.

Os valores de $\mathrm{pH}$ encontrados variaram de 4,17 a 4,60. Estes valores de $\mathrm{pH}$ estão abaixo ou bem próximos de 4,5 (valor que delimita o desenvolvimento de microrganismos). Desta forma, podem ser considerados como pós alimentícios ácidos de difícil ataque microbiano. Lima et al.[17] encontraram um valor de $\mathrm{pH}$ de 4,20 para a fibra do pendúculo de caju e para o suco de caju pH de 4,13, enquanto Felipe [13] obteve um $\mathrm{pH}$ de 3,49 para o pó do resíduo de maracujá (valor inferior ao encontrado neste trabalho). Os valores de sólidos solúveis totais ( ${ }^{\circ}$ Brix) encontrados nos pós alimentícios do bagaço de caju, bagaço de goiaba e casca de maracujá foram, respectivamente, 40,48; 10,48 e $20,56{ }^{\circ}$ Brix. Valores elevados eram previsíveis, tanto nos pós alimentícios obtidos das cascas quanto nos obtidos dos bagaços, uma vez que grande parte dos teores de sólidos solúveis totais presentes nas frutas, também estão presentes em seus resíduos. Esses teores podem variar com o processamento aplicado na extração da polpa, fatores climáticos, variedade do fruto, tipos de solo e diversos outros fatores agronômicos. Diversos autores, analisando polpas in natura destes frutos, encontraram o teor de sólidos solúveis totais para polpas de caju de 9,08 a $10,66^{\circ}$ Brix, para as de goiaba de 7,20 a 9,40 ${ }^{\circ}$ Brix e para as de maracujá de 13 a $15^{\circ}$ Brix. 
Tabela 1: Resultados das médias e desvio padrão dos parâmetros físico-químicos dos pós alimentícios dos resíduos do bagaço de caju, bagaço de goiaba e casca de maracujá.

\begin{tabular}{lccc}
\hline Parâmetros analisados & \multicolumn{3}{c}{ Pós alimentícios } \\
\cline { 2 - 4 } & Caju & Goiaba & Maracujá \\
\hline pH & $4,52 \pm 0,00$ & $4,60 \pm 0,08$ & $4,17 \pm 0,00$ \\
Umidade (\%) & $6,99 \pm 0,30$ & $3,33 \pm 0,11$ & $10,23 \pm 0,15$ \\
Lipídeos (\%) & $3,03 \pm 0,10$ & $9,74 \pm 0,10$ & $0,75 \pm 0,13$ \\
Proteína (\%) & $1,16 \pm 0,50$ & $1,16 \pm 0,46$ & $0,96 \pm 0,15$ \\
Sólidos solúveis totais ( ${ }^{\circ}$ Brix) & $40,48 \pm 0,00$ & $10,48 \pm 0,00$ & $20,56 \pm 0,00$ \\
Açúcares redutores (\% glicose) & $36,55 \pm 0,65$ & $5,31 \pm 0,13$ & $8,30 \pm 0,10$ \\
Açúcares totais (\% glicose) & $36,55 \pm 0,65$ & $5,31 \pm 0,13$ & $8,30 \pm 0,10$ \\
Acidez (g/100g) & $1,38 \pm 0,12$ & $1,21 \pm 0,16$ & $1,28 \pm 0,13$ \\
Vitamina C (mg/100g) & $34,72 \pm 0,29$ & $21,55 \pm 3,66$ & $11,76 \pm 0,13$ \\
Cinzas (\%) & $1,78 \pm 0,19$ & $2,14 \pm 0,32$ & $2,52 \pm 1,16$ \\
\hline
\end{tabular}

Normalmente, as frutas são bastante ricas em açúcares redutores (glicose e frutose), cuja determinação é feita para se avaliar a potencialidade de fermentação do produto. $\mathrm{Na}$ Tabela 1, observamos uma maior concentração de açúcares redutores no pó do bagaço do caju $(36,55 \%)$ e a menor quantidade foi encontrada no pó do bagaço da goiaba $(5,31 \%)$. Como sabemos, as frutas apresentam ausência ou pequenas concentrações de açúcar não-redutor, o que foi confirmado no presente trabalho. Ressaltamos que, com resíduos de frutas, a presença de açúcar não-redutor torna-se mais difícil.

Observando os resultados dos teores de lipídeos, verificamos que uma alta concentração foi encontrada no pó alimentício do resíduo de goiaba (9,74\%). Felipe [13], analisando os pós de resíduo de caju, goiaba e maracujá, obteve, respectivamente, os seguintes teores de lipídeos: 3,70, 14,05 e 1,18\%. Lima [16], em seu estudo do aproveitamento de bagaço de frutas tropicais, visando à extração de fibras e utilizando-se de bagaços de caju e abacaxi, oriundos do processamento de polpa, obteve valores para lipídeos de 1,26 e 0,17\%, respectivamente.

Nos valores encontrados na análise de proteína, verifica-se uma variação de 0,96 a $1,16 \%$ para os diferentes pós alimentícios analisados. Estes dados de proteína mostram que resíduos em pó não são fontes importantes de proteína. Os valores encontrados na análise de cinzas foram de $1,78 \%$ para o pó de resíduo de caju, $2,14 \%$ para o de goiaba e de $2,52 \%$ para o de maracujá, como é mostrado na Tabela 1. Estas altas taxas podem estar associadas a uma maior concentração dos minerais, presentes nos resíduos analisados, após o processo de extração da polpa e secagem. Felipe [13] encontrou para o pó de resíduo de caju $1,42 \%$, de goiaba $1,53 \%$ e de maracujá $3,32 \%$. No que se refere aos teores de cinzas, presentes na polpa in natura destas mesmas frutas, foram encontrados $0,33 \%$ para a polpa de caju, $1,39 \%$ para a polpa de goiaba e $0,40 \%$ para a polpa de maracujá (USDA [28]).

De acordo com os resultados encontrados na Tabela 1, verifica-se que o teor de umidade dos pós de resíduos de caju, de goiaba e de maracujá variou entre 3,33\% e $10,23 \%$. Felipe [13], ao analisar os mesmos pós, encontrou para o de caju um valor de umidade de $6,52 \%$, para o de goiaba $4,07 \%$ e para o de maracujá $9,14 \%$, valores bem próximos aos encontrados em nosso trabalho. Vale ressaltar que o teor de umidade do pó alimentício do resíduo de maracujá foi mais elevado que os outros. 
A Tabela 2 mostra os resultados das análises de fibra bruta e fibra alimentar, realizadas nos pós alimentícios dos resíduos de caju, goiaba e maracujá. Podemos observar que os pós alimentícios, obtidos do bagaço de caju, bagaço de goiaba e casca de maracujá, apresentaram os seguintes teores de fibra bruta: 9,92; 39,56 e 26,31\%. Estes valores de fibra bruta são superiores aos encontrados pelo
ENDEF [11] nas frutas in natura do caju, da goiaba e do maracujá, que foram, respectivamente, de 1,$5 ; 5,3$ e $0,70 \%$. Felipe [13], analisando pós de resíduos de frutas tropicais, apresentou o teor de fibra bruta para o bagaço de caju de $5,91 \%$, para o bagaço de goiaba de $40,98 \%$ e para a casca de maracujá de $20,70 \%$, valores bem próximos aos encontrados em nosso estudo.

Tabela 2: Resultados das médias e desvio padrão dos teores de fibra bruta e alimentar dos pós de resíduos do bagaço de caju, bagaço de goiaba e casca de maracujá.

\begin{tabular}{lccc}
\hline Parâmetros analisados $(\%$ & \multicolumn{3}{c}{ Pós alimentícios } \\
\cline { 2 - 4 } em base seca) & Caju & Goiaba & Maracujá \\
Fibra bruta (\%) & $9,92 \pm 1,28$ & $39,56 \pm 0,40$ & $26,31 \pm 1,09$ \\
Fibra alimentar (\%) & $3,26 \pm 0,01$ & $24,46 \pm 3,73$ & $17,07 \pm 0,79$ \\
\hline
\end{tabular}

O teor de fibra alimentar encontrado nas amostras analisadas foi de: $3,26 \%$ para o bagaço de caju, de $24,46 \%$ para o bagaço da goiaba e de $17,07 \%$ para a casca de maracujá. $\mathrm{O}$ teor de fibra alimentar do bagaço de caju, bagaço da goiaba e de casca do maracujá representam, respectivamente, 32,96; 61,83 e $64,88 \%$ da fibra bruta total. Estes resultados mostram que os pós de goiaba e maracujá são importantes em relação à quantidade de fibra alimentar. Um alimento com teor de 2 a $3 \%$ pode ser considerado uma boa fonte de fibra alimentar. Segundo o regulamento técnico, referente à informação nutricional complementar (Portaria nọ 27), o alimento pode ser considerado fonte de fibra alimentar quando apresenta, no produto acabado, $3 \mathrm{~g} / 100 \mathrm{~g}$ (base integral) para alimentos sólidos e $1,5 \mathrm{~g} / 100 \mathrm{ml}$ (base integral) para líquidos. Com o dobro deste conteúdo, pode ser tratado como alimento de elevado teor de fibra alimentar (Brasil [6]).

Considerando os resultados obtidos neste trabalho, pode-se afirmar que o bagaço de caju, o bagaço de goiaba e a casca do maracujá apresentam alto índice de fibra alimentar. Tal fato sugere que novos produtos a base de fibra, obtidos dos resíduos destas frutas, podem ser formulados para prevenir doenças, principalmente relacionadas ao trato gastrointestinal e ao coração. Pode-se, também, devido ao alto teor de fibra alimentar desses pós alimentícios, apontá-los como alternativas para serem utilizados em vários produtos, como os de panificação (biscoitos, pães e massas alimentícias), amplamente consumidos pela população.

Vários trabalhos têm mostrado a importância das fibras nos alimentos. Kan et al [15] estudaram as ações benéficas da fibra alimentar em doenças respiratórias e função pulmonar. Silva et al.[26] estudaram a farinha de jatobá como fonte de fibra para ser adicionada a biscoitos do tipo cookies, tendo apresentado, para a fibra insolúvel, um percentual de 36,4\%. Perez e Germani [21], analisando farinha obtida de berinjela, encontrou $44,12 \%$ de fibra alimentar total. Em seu estudo, a adição de farinha de berinjela promoveu alteração significativa no teor de fibra alimentar total das farinhas mistas com farinha de trigo, ocorrendo acréscimo gradativo a medida que se aumentou o teor de farinha de berinjela. Ferreira et al.[14] encontraram um teor de fibras médio de 58,98 $\mathrm{g} / 100 \mathrm{~g}$ para semente de maracujá, valor próximo ao encontrado por Córdova et al. [8] de 57,32 g/100g na casca do maracujá. 


\section{Conclusões}

A partir dos resultados obtidos, podese considerar que os pós alimentícios dos resíduos de caju, goiaba e maracujá são fontes importantes de vitamina $\mathrm{C}$ e apresentam alto índice de fibra bruta e alimentar.

Os pós alimentícios de caju, goiaba e maracujá apresentaram altos teores de sólidos solúveis e baixos teores de proteína. No que se refere aos lipídeos, o pó alimentício obtido de resíduo de goiaba foi o que apresentou o maior teor, que corresponde a $9,74 \%$.

Os pós alimentícios, obtidos de resíduos de frutas, são ricos em fibras e outros componentes, portanto, podem ser aproveitados na formulação de novos produtos alimentícios (biscoitos, bolachas, pães,sopas...etc.)

\section{Agradecimentos}

À Fundação Cearense de Amparo a Pesquisa - FUNCAP pela ajuda financeira a este trabalho, através de uma bolsa de estudo.

\section{Referências Bibliográficas}

[1] A.A.C.C. - American Association of Cereal Chemists. Approved Methods of American Association of Cereal Chemists, 9 ed. St. Paul: AACC, 1995, V.1 e 2.

[2] A.O.A.C. - Association of Official Analytical Chemists. Official Methods of Analysis of the Association of Analytical Chemistry. 12ed. Washington, 1995.

[3] Anuário brasileiro da fruticultura 2006. Gilson R. da Rosa. [et al.].- Santa Cruz do Sul: Editora Gazeta Santa Cruz, 2006. 136p: il.

[4] Beling, R.R. Anuário Brasileiro de Fruticultura. Santa Cruz do Sul: Editora Gazeta Santa Cruz, 2005.

[5] Brasil. Ministério da Saúde. Secretaria de Vigilância Sanitária. Portaria na33, de 13 de janeiro de 1998, adota valores para ingestão diária recomendada (IDR) de vitaminas, minerais e proteínas. Diário Oficial da República Federativa do Brasil. Brasília -DF, de 16 de janeiro de 1998.

[6] Brasil. Ministério da Saúde - Agência Nacional de Vigilância Sanitária. Portaria $n^{\circ}$ 27 de 13/01/1998 - Regulamento Técnico Referente à Informação Nutricional Complementar.

[7] Brasil. Ministério da Saúde. Agência Nacional de Vigilância Sanitária. Métodos Físico-Químicos para Análise de Alimentos. Diário Oficial da União Brasília: Ministério da Saúde, 2005. 1018p.
[8] Córdova KV, Gama, TMMTB, Winter GMG, Freitas RJS. Características físicoquímicas da casca do maracujá amarelo (Passiflora edulis flavicarpa degener) obtida por secagem. Boletim Ceppa, Curitiba, v. 23. n. 2, p. 221-230, jan/ jun. 2005.

[9] Dutra OJE, Marchini JS. Ciências Nutricionais. São Paulo: Sarvier, 1998.

[10] Embrapa - Empresa Brasileira de Pesquisa e Agropecuária. Iniciando um pequeno grande negócio agroindustrial: polpa e suco de frutas/ Embrapa Agroindústria de Alimentos, Serviço Brasileiro de Apoio às Micro e Pequenas Empresas - Brasília: Embrapa Informação Tecnológica, 2003. 123p. - (Série agronegócios).

[11] ENDEF/ IBGE. Estudo Nacional da Despesa Familiar. Instituto Brasileiro de Geografia e Estatística. Tabela de Composição de Alimentos. $5^{\text {a }}$ ed. Rio de Janeiro: IBGE, 1999.

[12] FAO (Food and Agriculture Organization of United Nations). Faostat. Fao Statistics

Division 2006. Disponível em:

<http://faostat.fao.org/site/408/DesktopDefault .aspx?PageID=408>. Acesso em: 18 out. 2006.

[13] Felipe EMF. Caracterização FísicoQuímica de Pós Alimentícios Obtidos de Resíduos de Frutas Tropicais. [Dissertação] Fortaleza: Universidade Federal do Ceará, 2006. 93p.

[14] Ferreira RA, Colussi F, Ayub RA. Caracterização de subprodutos da 
industrialização do maracujá - aproveitamento das sementes. Revista Brasileira de Fruticultura, 2004; 26 (1):101-102.

[15] Kan H, Stevens J, Heiss G, Rose KM, Londres, SJ. Dietary fiber, lung function, and chronic obstructive pulmonary disease in the atherosclerosis risk in communities study. American Journal of Epidemiology. 2008; 167 (5): 570-578.

[16] Lima LMO. Estudo do aproveitamento dos bagaços de frutas tropicais, visando a extração de fibras. [Dissertação] Natal: Universidade Federal do Rio Grande do Norte. 2001.102p.

[17] Lima AC, García NHP, Lima JR. Obtenção e caracterização dos principais produtos do caju. Boletim Ceppa. 2004; 22(1): 133-144.

[18] Lobo AR, Silva GML. Implicações Nutricionais no Consumo de Fibras e Amido Resistente. Nutrição em Pauta. Janeiro/ fevereiro, 2001.

[19] Oliveira MEB, Feitosa T, Bastos, MSR, Silva MGG, Branco MAAC. Polpas

Congeladas de Frutas - Avaliação da

qualidade em quatro Estados do Nordeste

Brasileiro. In: Congresso Brasileiro de Ciência e Tecnologia De Alimentos, Fortaleza, Ceará: SBCTA, 1998. Anais... Ceará, 1998.

[20] Pearson D, Cox HE. The chemical analysis of foods. New York: Chem. Publ. 1976.

[21] Perez PMP, Germani R. Farinha mista de trigo e berinjela: características físicas e químicas. Boletim Ceppa. 2004; 22(1): 15-24.

[22] Petinari RA, Tarsitano MAA. Comercialização de caju in natura na região Noroeste do Estado de São Paulo: Revista
Brasileira de Fruticultura. 2002; 24 (3): 697699.

[23] Prosky L, Asp N, Schweizer TF, Devries JW, Furda I. Determination of insoluble, soluble, and total dietary fiber in foods and food products: interlaboratory study. Journal of the Association Official Analytical Chemists. 1988; 71 (5); 1017-1023.

[24] Sandi D, Chaves JBP, Souza ACG, Silva MTC, Parreiras JFM. Correlações entre características físico-químicas e sensoriais em suco de maracujá-amarelo (Passiflora edulis VAR. flavicarpa ) durante o armazenamento. Ciência de Tecnologia e Alimentos. 2003; 23(3); 355-361.

[25] SEAGRE - Secretaria de Agricultura, Irrigação $E$ Reforma Agrária. Goiaba e Maracujá. Obtido via Internet. <http://www.seagri.ce.gov.br>. 2002. Acesso em 21/12/2003.

[26] Silva MR, Silva MAAP, Chang YK. Utilização da farinha de jatobá (Hymenaea stigonocarpa Mart.) na elaboração de biscoitos tipo cookie e avaliação de aceitação por testes sensoriais afetivos univariados e multivariados IN: Ciência e Tecnologia de Alimentos. 1998; 18 (1): 25-34.

[27] Silva DS. Estabilidade de suco tropical de goiaba (Psidium guajava L.) não-adoçado obtido pelos processos de Enchimento a quente e asséptico. [Dissertação]. Fortaleza: Universidade Federal do Ceará; 2007. 98p.

[28] USDA - Department of Agriculture (USDA). Agricultural Research Service: USDA Nutrient Database for Standard Reference, release 17, SR 17 [database on line]. 2004. Available from 〈www.nal.usda.gov/fnic/foodcomp >. Acessed $31 / 10 / 2004$

\section{Autores}

Ana Maria Athayde Uchoa - Mestranda, Departamento de Tecnologia de Alimentos - CCA/UFC. Jose Maria Correia da Costa - Professor Doutor, Departamento de Tecnologia de Alimentos, CCA/UFC.

Correspondência: Av.Mister Hull, 2970- 60356-000 - Fortaleza/CE, Tel: (85) 3366-9740, email: correia@ufc.br.

Geraldo Arraes Maia - Professor Doutor, Departamento de Tecnologia de Alimentos, CCA/UFC.

Elisabeth Mary Cunha Silva - Professora Doutora, Departamento de Tecnologia de Alimentos, CCA/UFC. Ana de Fátima Urano Carvalho - Professora Doutora, Departamento de Biologia, CC/UFC.

Tatyane Ribeiro Meira - Estudante de Engenharia de Alimentos - Bolsista IC, Departamento de Tecnologia de Alimentos CCA/UFC.

Recebido em: 03/10/2007

Aceito em: 15/07/2008 